\title{
Flow of Brinkman Fluid with Heat Generation and Chemical Reaction
}

\author{
M. Ramzan, ${ }^{1}$ Zaib Un Nisa, ${ }^{2}$ M. Ahmad, ${ }^{1}$ and M. Nazar $\mathbb{D}^{1,3}$ \\ ${ }^{1}$ Centre for Advanced Studies in Pure and Applied Mathematics, Bahauddin Zakariya University, Multan, Pakistan \\ ${ }^{2}$ Department of Mathematics, University of Education Lahore, Multan Campus, Multan, Pakistan \\ ${ }^{3}$ School of Mathematical Sciences, University of Science and Technology of China, Hefei, Anhui, China
}

Correspondence should be addressed to M. Nazar; mudassar_666@yahoo.com

Received 8 June 2021; Accepted 7 August 2021; Published 25 August 2021

Academic Editor: Ali Akgül

Copyright $\odot 2021$ M. Ramzan et al. This is an open access article distributed under the Creative Commons Attribution License, which permits unrestricted use, distribution, and reproduction in any medium, provided the original work is properly cited.

Unsteady magnetohydrodynamics (MHD) flow of fractionalized Brinkman-type fluid over a vertical plate is discussed. In the model of problem, additional effects such as heat generation/absorption and chemical reaction are also considered. The model is solved by using the Caputo fractional derivative. The governing dimensionless equations for velocity, concentration, and temperature profiles are solved using the Laplace transform method and compared graphically. The effects of different parameters like fractional parameter, heat generation/absorption $Q$, chemical reaction $R$, and magnetic parameter $M$ are discussed through numerous graphs. Furthermore, comparison among ordinary and fractionalized velocity fields are also drawn. From the figures, it is observed that chemical reaction and magnetic field have decreasing effect on velocity profile, whereas thermal radiation and mass Grashof numbers have increasing effect on the velocity of the fluid.

\section{Introduction}

The important significance of non-Newtonian fluids can be seen in applied mathematics, engineering, and physics. It has various significances in many areas, such as uses of lubricants, biological fluid food processing, or plastic manufacturing. Some commonly examples of non-Newtonian fluids are custard, colloids, melted butter, paint, ketchup, starch suspensions, blood, toothpaste, gels, shampoo, and corn starch.

Mass transfer and heat transfer occurs mostly in nature due to temperature and concentration differences, respectively. Today, research work in magnetohydrodynamics (MHD) has substantial significance as these flows are absolutely prevailing in nature.

Convection flow with porous media has numerous applications such as flows in soils, solar power collectors, heat transfer correlated with geothermal systems, heat source in the field of agricultural storage system, heat transfer in nuclear reactors, heat transfer in aerobic and anaerobic reactions, heat evacuation from nuclear fuel detritus, and heat exchangers for porous material.
MHD fluid has many implementations in meteorology, distillation of gasoline, energy generators, geophysics, accelerators, petroleum industry, astrophysics, polymer technology, aerodynamics, and boundary layer control and in material processes such as glass fiber drawing, extrusion, and casting wire. The flow of viscous fluid through a perpendicular plate is analyzed by Swamy et al. [1]. The effect of mass diffusion on MHD fluid with porosity has been observed by Chaudhary et al. [2]. Exact solution for magnetohydrodynamics flow through a perpendicular plate in the existence of porosity is obtained by Sivaiah et al. [3]. The solution for unsteady flow of viscous fluid with porosity is obtained by Das and Jana [4].

Furthermore, convection flow in the existence of porosity has wide applications such as ground water hydrology, oil extraction, geothermal systems, cooling systems, storage of nuclear waste materials, energy-efficient drying processes, solid matrix heat exchangers, and wall-cooled catalytic reactors. Kataria and Patel [5] analyzed the impact of magnetic field with heat transfer over a plate. The authors of [6] discussed the solution of viscous fluid flow with thermal 
radiation. Chamkha [7] discussed the effect of heat source on MHD fluid through a moving plate.

The authors of [8] studied the flow of polar fluid through a plate. Rahman and Sattar [9] studied the flow of fluid with a heat source. Rajesh and Varma [10] studied the influence of mass diffusion on magnetohydrodynamic fluid flow. The authors of [11] analyzed the solution of convection flow through a vertical plate. They also discussed the solution for time-dependent concentration and temperature. Convection flow immersed in a porous media through a surface is discussed in [12-14].

The impact of conjugate flow of MHD fluid is discussed by Khan et al. [15]. Rajesh et al. [16] discussed the MHD flow through a moving plate. MHD flow through an accelerated surface in the existence of porous media is discussed by Chaudhary et al. [17]. The authors also analyzed the solution of velocity field graphically. Das [18] analyzed the solution of magnetohydrodynamics of convection flow through a plate. Pal et al. [19] examined the solution of viscous fluid with thermal radiation on magnetohydrodynamics flow, whereas the solution for convection flow with nonuniform temperature through a moving plate is obtained by Seth et al. [20]. The solution of nanofluid with ramped temperature is studied by Khalid et al. [21].

The discussion of mass diffusion has empirical use in numerous areas of engineering and applied sciences. These phenomena play a vital role in cooling of a nuclear reactor and tabular reactor, chemical industry, mixture of terracotta material, petroleum industry, and decomposition of rigid materials. Seddeek et al. [22] examined the MHD fluid flow with thermal radiation. An intensive study of chemical reaction with heat source/sink is studied by Shah et al. [23]. Seth et al. [24] obtained the solution of unsteady magnetohydrodynamic flow of the fluid over a plate with ramped condition. The solution of convection flow of MHD fluid over a plate with heat generation/absorption is obtained by Shateyi and Motsa[25]. MHD fluid flow with Ohmic heating and heat generation is analyzed by Kasim et al. [26]. The exact solution of MHD fluid with mass transfer immersed in a porous media is discussed by Ali et al. [27]. The exact solution of magnetohydrodynamic flow of a Brinkman fluid perpendicular to the plate is analyzed by Khan et al. [28]. The analytical investigation of Brinkman fluid flow with variable concentration, temperature, and velocity is obtained by Ali et al. [29]. The flow of nanofluid with thermal radiation is studied in [30-35]. Patel et al. [36] studied the effect of Joule's heating on ferrofluid. The influence of Brownian motion and thermophoresis is studied by Mittal and Kataria [37]. Kataria [38] studied the effect of radiation and magnetic field on Casson fluid. Hashemi et al. [39] analyzed the solution of a circular rod. Some flows of fluids with numerical and computational methods are discussed in [40-42].

In this problem, the model of unsteady magnetohydrodynamic free convection flow of Brinkman fluid through a plate is considered. The impact of chemical reaction and heat absorption/generation is added into account. Firstly, the governing equations have been made nondimensional and then solved semianalytically. The results for velocity profile, temperature profile, and concentration profile are obtained and then analyzed graphically. Various graphs are plotted and discussed for different parameters, which are used in the flow model. The comparison between ordinary and fractionalized fluid is drawn graphically and shows that Caputo fractional derivative is the best choice for controlled fluid velocity.

\section{Mathematical Description of the Model}

The magnetohydrodynamic flow of Brinkman fluid through a plate with mass and heat transfer is considered. The fluid is flowing along the $x$ axis. The motion of fluid depends on $y$-axis and time $t_{1}$. The plate and fluid have concentration $C_{\infty}$ and temperature $T_{\infty}^{*}$ at constant $t_{1}^{*}=0$ with zero velocity. But, for $t_{1}>0$, the plate starts to move in the plane with uniform velocity $U_{1} e^{a t}{ }_{1}$. The concentration and temperature of the plate increased linearly to $C_{w}$ and $T_{w}$ with time $t$. A constant strength $\beta_{0}$ of magnetic field is applied normally. In view of the above assumption and using Boussinesq's approximation, the convection flow of Brinkman fluid with chemical reaction, and magnetic field through a plate, the linear momentum equation is

$$
\begin{aligned}
\rho \frac{\partial u_{1}\left(\dot{y}, t_{1}^{\prime}\right)}{\partial t_{1}^{\prime}} & +B_{1} u_{1}\left(\dot{y}, t_{1}^{\prime}\right)=\frac{\partial \tau\left(\dot{y}, t_{1}^{\prime}\right)}{\partial y^{\prime}}+g \beta_{T^{*}}\left(T^{*}-T_{\infty}^{*}\right) \\
& -\sigma \beta_{0}^{2} u_{1}\left(y^{\prime}, t_{1}^{\prime}\right)+g \beta_{C}\left(C^{*}-C_{\infty}^{*}\right) .
\end{aligned}
$$

Shear stress $\tau$ is

$$
\tau=\mu \frac{\partial u_{1}\left(y, t_{1}\right)}{\partial y} .
$$

Thermal equation is

$$
\rho C_{p} \frac{\partial T\left(y, t_{1}^{\prime}\right)}{\partial t_{1}}=-\frac{\partial q_{1}\left(\dot{y}, t_{1}^{\prime}\right)}{\partial y^{\prime}}+Q_{1}\left(T-T_{\infty}\right) .
$$

According to Fourier's Law, $q_{1}\left(y, t_{1}^{\prime}\right)$ is given by

$$
q_{1}\left(y^{\prime}, t_{1}^{\prime}\right)=-\alpha_{0} \frac{\partial T\left(\dot{y}, t_{1}^{\prime}\right)}{\partial y^{\prime}} .
$$

Diffusion equation is

$$
\frac{\partial C^{\prime}\left(y^{\prime}, t_{1}^{\prime}\right)}{\partial t_{1}^{\prime}}=-\frac{\partial J_{1}\left(y^{\prime}, t_{1}^{\prime}\right)}{\partial y^{\prime}}-R_{1}\left(C^{\cdot}-C_{\infty}^{*}\right) .
$$

According to Fick's Law, $J_{1}\left(y^{\prime}, t_{1}^{\prime}\right)$ is given by

$$
J_{1}\left(\dot{y}, t_{1}^{\prime}\right)=-D_{m} \frac{\partial C\left(y, t_{1}^{\prime}\right)}{\partial y^{\prime}}
$$

The boundary conditions for the flow model are 


$$
\begin{aligned}
u_{1}\left(y^{\prime}, t_{1}^{\prime}\right) & =0, \\
T^{\prime}\left(y^{\prime}, t_{1}^{\prime}\right) & =T_{\infty}^{\cdot}, \\
C^{\prime}\left(y^{\prime}, t_{1}\right) & =C_{\infty}, \\
y^{\prime}>0, t_{1} & =0 . \\
u_{1}\left(0, t_{1}^{\prime}\right) & =U_{1} f\left(t_{1}^{\prime}\right)=U_{1} e^{a t_{1}}, \\
T^{\prime}\left(0, t_{1}^{\prime}\right) & =T_{w}, \\
C^{\prime}\left(0, t_{1}^{\prime}\right) & =C_{w}^{\prime}, \\
t_{1} & >0, \\
u_{1}\left(y^{\prime}, t_{1}^{\prime}\right) & \longrightarrow 0, \\
T^{\prime}\left(y^{\prime}, t_{1}^{\prime}\right) & \longrightarrow 0, \\
C^{\prime}\left(y^{\prime}, t_{1}^{\prime}\right) & \longrightarrow 0, \\
y^{\prime} & \longrightarrow \infty, t_{1}>0 .
\end{aligned}
$$

To write the flow model in dimensionless form, we used the following dimensionless variables:

$$
\begin{aligned}
y^{*} & =\frac{U y}{v}, \\
t^{*} & =\frac{U^{2} t_{1}}{v}, \\
\operatorname{Pr}^{*} & =\frac{\rho \nu C_{p}}{\alpha_{0}}, \\
v^{*} & =\frac{u_{1}}{U}, \\
\mathrm{Gr}^{*} & =\frac{\rho \nu \beta_{T} \cdot\left(T_{w}^{*}-T_{\infty}^{*}\right)}{U^{3}}, \\
M^{*} & =\frac{\beta_{0}^{2} \sigma}{\rho U^{2}}, \\
Q^{*} & =\frac{Q_{1} \nu}{U^{2} \rho C_{p}}, \\
R^{*} & =\frac{R_{1} v}{U^{2}}, \\
C^{*} & =\frac{C^{*}-C_{\infty}^{*}}{C_{w}^{\prime}-C_{\infty}^{*}}, \\
\mathrm{Sc}^{*} & =\frac{v}{D_{1}} \mathrm{Gm}^{*}=\frac{\rho v \beta_{C}\left(C_{w}^{*}-C_{\infty}^{*}\right)}{U^{3}} .
\end{aligned}
$$

Using nondimensional variables from equations in (10) into the equations (1)-(9), we have

$$
\begin{aligned}
\left(\frac{\partial}{\partial t}+B\right) v(y, t)= & K_{1} \frac{\partial \tau}{\partial y}-M v(y, t)+\operatorname{Gr} T(y, t) \\
& +\operatorname{GmC}(y, t), \\
\tau= & L_{1} \frac{\partial v(y, t)}{\partial y},
\end{aligned}
$$

$$
\begin{gathered}
\frac{\partial T(y, t)}{\partial t}+K_{2} \frac{\partial q}{\partial y}-Q T(y, t)=0, \\
q=-m_{1} \frac{\partial T(y, t)}{\partial y}, \\
\frac{\partial C(y, t)}{\partial t}+K_{3} \frac{\partial J}{\partial y}+R C(y, t)=0, \\
J=-n_{1} \frac{\partial C(y, t)}{\partial y},
\end{gathered}
$$

with boundary conditions as

$$
\begin{aligned}
v(y, 0) & =T(y, 0)=C(y, 0)=0, \quad y>0, t=0, \\
v(y, t) & =e^{a t}, T(y, t)=1, C(y, t)=1, \quad t>0, \\
v(\infty, t) & =T(\infty, t)=C(\infty, t) \longrightarrow 0, \quad t>0,
\end{aligned}
$$

where

$$
\begin{aligned}
& K_{1}=\frac{\tau}{U^{2} \rho}, \\
& K_{2}=\frac{q}{U \rho C_{p}\left(T_{w}-T_{\infty}\right)}, \\
& K_{3}=\frac{J_{1}}{\left(C_{w}-C_{\infty}\right) U}, \\
& L_{1}=\frac{U^{2} \mu}{q_{1} v}, \\
& m_{1}=\frac{\alpha_{0}\left(T_{w}-T_{\infty}\right) U}{q v}, \\
& n_{1}=\frac{D_{m}\left(C_{w}-C_{\infty}\right) U}{J v},
\end{aligned}
$$

where $\mathrm{Gr}, B, \mathrm{Sc}, Q, M, \mathrm{Pr}, \mathrm{Gm}$, and $v$ represent the Grashof number for heat transfer, Brinkman parameter, Schmidt number, nondimensional heat source, magnetic field, Prandtl number, mass Grashof number, and velocity of the fluid, respectively.

\section{Generalized Model}

Equation (12) is frictionally generalized by Blair and Caffyn [43]:

$$
\tau=L_{1-\beta} D_{t}^{1-\beta} \frac{\partial u(y, t)}{\partial y}, \quad 1 \geq \beta>0 .
$$

Equation (14) is generalized by using Fourier Law defined by Povstenko and Hristov [44, 45]:

$$
q=-m_{1-\gamma} D_{t}^{1-\gamma} \frac{\partial T(y, t)}{\partial y}, \quad 1 \geq \gamma>0 .
$$

By using Fick's Law, equation (16) is generalized as 


$$
J=-n_{1-\alpha} D_{t}^{1-\alpha} \frac{\partial C(y, t)}{\partial y}, \quad 1 \geq \alpha>0 .
$$

Using equation (19) into equation (11), equation (20) into equation (13), and equation (21) into equation (15), we have

$$
\begin{aligned}
{\left[\frac{\partial}{\partial t}+B\right] v(y, t) } & =K_{1} \frac{\partial}{\partial y}\left[L_{1-\beta} D_{t}^{1-\beta} \frac{\partial v(y, t)}{\partial y}\right]-M v(y, t)+\operatorname{Gr} T(y, t)+\operatorname{GmC}(y, t), \\
\frac{\partial T(y, t)}{\partial t} & =K_{2} \frac{\partial}{\partial y}\left[m_{1-\gamma} D_{t}^{1-\gamma} \frac{\partial T(y, t)}{\partial y}\right]+Q T(y, t), \\
\frac{\partial C(y, t)}{\partial t} & =K_{3} \frac{\partial}{\partial y}\left[n_{1-\alpha} D_{t}^{1-\alpha} \frac{\partial C(y, t)}{\partial y}\right]-R C(y, t) .
\end{aligned}
$$

Taking inversion left operator in equations (22)-(24), we obtain

$$
\begin{aligned}
{\left[I_{t}^{1-\beta} \frac{\partial}{\partial t}+B I_{t}^{1-\beta}\right] v(y, t)=} & {\left[D_{t}^{\beta}+B I_{t}^{1-\beta}\right] v(y, t)=P_{1} \frac{\partial^{2} v(y, t)}{\partial y^{2}}-} \\
& I_{t}^{1-\beta} M v(y, t)+I_{t}^{1-\beta} \operatorname{Gr} T(y, t)+I_{t}^{1-\beta} \operatorname{GmC}(y, t), \\
I_{t}^{1-\gamma} \frac{\partial T(y, t)}{\partial t}= & D_{t}^{\gamma} T(y, t)=P_{2} \frac{\partial^{2} T(y, t)}{\partial y^{2}}+Q I_{t}^{1-\gamma} T(y, t), \\
I_{t}^{1-\alpha} \frac{\partial C(y, t)}{\partial t}= & D_{t}^{\alpha} C(y, t)=P_{3} \frac{\partial^{2} C(y, t)}{\partial y^{2}}-R I_{t}^{1-\alpha} C(y, t),
\end{aligned}
$$

where $P_{1}=K_{1} L_{1-\beta}=1$ when $\beta \longrightarrow 1, P_{2}=K_{2} m_{1-\gamma}=1 / \operatorname{Pr}$ when $\gamma \longrightarrow 1, P_{3}=K_{3} n_{1-\alpha}=1 / \mathrm{Sc}$ when $\alpha \longrightarrow 1$, and $D_{t}^{\alpha} v(y, t)$ represents the Caputo fractional derivative of $v(y, t)$ as

$$
D_{t}^{\alpha} v(y, t)= \begin{cases}\frac{1}{\Gamma(1-\alpha)} \int_{0}^{t} \frac{1}{(t-q)^{\alpha}} \frac{\partial v(y, q)}{\partial q} \mathrm{~d} q, & 0 \leq \alpha<1, \\ \frac{\partial v(y, t)}{\partial t}, & \alpha=1,\end{cases}
$$

and the left inverse operator of the derivative operator is

$$
I_{t}^{\alpha} g(y, t)=\frac{1}{\Gamma(\alpha)} \int_{0}^{t} g(y, s)(t-s)^{\alpha-1} \mathrm{~d} s,
$$

with

$$
I_{t}^{1-\alpha} \frac{\partial g(y, t)}{\partial t}=D_{t}^{\alpha} g(y, t) .
$$

\section{Solution of the Problem}

Equations (25)-(27) with initial and boundary conditions are solved semianalytically.
4.1. Calculation of Concentration. Solution of equation (27) is

$$
s^{\alpha} \bar{C}(y, s)=P_{3} \frac{\partial^{2} \bar{C}(y, s)}{\partial y^{2}}-R \frac{\bar{C}(y, s)}{s^{1-\alpha}} .
$$

Boundary conditions satisfying equation (31) are

$$
\begin{aligned}
\bar{C}(0, s) & =s^{-1}, \\
\bar{C}(y, s) & \longrightarrow 0, \\
y & \longrightarrow \infty
\end{aligned}
$$

Equation (31) is solved by using conditions given in equation (32), and we have

$$
\bar{C}(y, s)=s^{-1} e^{-y \sqrt{\left(1 / P_{3}\right)\left((s+R) / s^{1-\alpha}\right)}},
$$

which is complicated and cannot be solved analytically. The numerical result of equation (33) is obtained by using the algorithm in [46, 47].

4.2. Calculation of Temperature. Solution of equation (26) is

$$
s^{\gamma} \bar{T}(y, s)=P_{2} \frac{\partial^{2} \bar{T}(y, s)}{\partial y^{2}}+Q \frac{\bar{T}(y, s)}{s^{1-\gamma}} .
$$


Boundary conditions satisfying equation (34) are

$$
\begin{aligned}
\bar{T}(0, s) & =s^{-1}, \\
\bar{T}(y, s) & \longrightarrow 0, \\
y & \longrightarrow \infty .
\end{aligned}
$$

Equation (34) is solved by using conditions given in equation (35), which results in

$$
\bar{T}(y, s)=\frac{1}{s} e^{-y \sqrt{\left(1 / P_{2}\right)\left((s-Q) / s^{1-\gamma}\right)}},
$$

which is complicated one and cannot be solved analytically. The numerical result of equation (36) is obtained by using the algorithm in $[46,47]$.

\subsection{Calculation of Velocity. Solution of equation (25) is}

$$
\begin{aligned}
{\left[s^{\beta}+\frac{B}{s^{1-\beta}}\right] v(y, t)=} & P_{1} \frac{\partial^{2} v(y, t)}{\partial y^{2}}-\frac{1}{s^{1-\beta}} M v(y, t) \\
& +\frac{1}{s^{1-\beta}} \operatorname{Gr} T(y, t)+\frac{1}{s^{1-\beta}} \operatorname{GmC}(y, t) .
\end{aligned}
$$

Boundary conditions satisfying equation (37) are

$$
\begin{aligned}
\bar{v}(0, s) & =\frac{1}{s-a}, \\
\bar{v}(y, s) & \longrightarrow 0, \\
y & \longrightarrow \infty .
\end{aligned}
$$

Equation (37) is solved by using conditions given in equation (38), and we obtain

$$
\begin{aligned}
\bar{v}(y, s)= & \frac{1}{s-a} e^{-y \sqrt{\left(1 / P_{1}\right)\left((s+H) / s^{1-\beta}\right)}}+\frac{\mathrm{Gr}}{s\left(s^{1-\beta}\right)\left[\left(P_{1} / P_{2}\right)\left((s-Q) / s^{1-\gamma}\right)-\left((s+H) / s^{1-\beta}\right)\right]} \\
& {\left[e^{-y \sqrt{\left(1 / P_{1}\right)\left((s+H) / s^{1-\beta}\right)}}-e^{-y \sqrt{\left(1 / P_{2}\right)\left((s-Q) / s^{-\gamma}\right)}}\right]+\frac{\mathrm{Gm}}{s\left(s^{1-\beta}\right)\left[\left(P_{1} / P_{3}\right)\left((s+R) / s^{1-\alpha}\right)-\left((s+H) / s^{1-\beta}\right)\right]} } \\
& {\left[e^{-y \sqrt{\left(1 / P_{1}\right)\left((s+H) / s^{1-\beta}\right)}}-e^{-y \sqrt{\left(1 / P_{3}\right)\left((s+R) / s^{1-\alpha}\right)}}\right] }
\end{aligned}
$$

which is much complicated, so it cannot be solved analytically. Numerical result of equation (39) will be obtained by using the algorithm in [46, 47].

\section{Results and Discussion}

Semianalytical solution for MHD flow of Brinkman fluid with a combined concentration and temperature gradient over a plate is obtained. The generalized model is solved with a Caputo fractional derivative. The graph of concentration profile, temperature profile, and velocity profile are plotted for different parameters.

Figure 1 represents the effect of $B$ on $v(y, t)$. It is noted that the $v(y, t)$ decreases with increasing values of the Brinkman parameter. Physically, Brinkman is the relation between drag force and density; therefore, drag force increases with increasing values of the Brinkman parameter which decays down the fluid motion. The behavior of $\mathrm{Gm}$ is reported in Figure 2. From this graph, it is concluded that the magnitude of fluid velocity rises by raising the values of $\mathrm{Gm}$. $\mathrm{Gm}$ is the relative strength of viscous force and concentration buoyancy force. As $\mathrm{Gm}$ increases, the motion of fluid is accelerated due to an increment of buoyancy force. Figure 3 represents the impact of different values of $\mathrm{Gr}$ on $v(y, t)$. From this graph, it is noted that velocity distribution is directly proportional with Gr. Physically, Gr is a relation between viscous force and buoyancy force. Therefore, with an increment in the values of $\mathrm{Gr}$, buoyancy force is increased which raises the magnitude of $v(y, t)$.

Figure 4 displays the behavior of $M$ and $Q$ on fluid motion. The speed of fluid layer is reduced for raising values of $M$ as shown in the graph. Physically, the Lorentz force creates the low resistivity which increases the thickness or width of momentum boundary layer in the solution. The impact of $Q$ on fluid velocity is displayed in Figure 4. The $v(y, t)$ is increased by increasing values of $Q$ as depicted in the figure. Physically, when the values of heat generation parameter are increased, the thermal conductivity becomes dominant. Fluid particles attract each other weakly which improve the fluid motion.

Figure 5 represents the behavior of $R$ and $\operatorname{Pr}$ on the $v(y, t)$. The graph shows that $v(y, t)$ falls down for larger values of $R$. The impact of various values of $\operatorname{Pr}$ on $v(y, t)$ is displayed in Figure 5. Pr represents the ratio of momentum (product of mass and velocity) diffusion to thermal diffusion. In the problems of heat transfer, Pr manages the thickness of boundary layer and momentum (velocity). For larger value of Pr, diffusion of heat becomes slow as compared to the fluid momentum (velocity) which decreases the thermal conductivity (thickness) and raises the boundary layer momentum. The influence of $R$ on fluid motion is shown in Figure 5. From the figure, it is concluded that fluid motion decays by increasing the values of $R$. Physically, boundary layer thickness is increased by increasing values of 

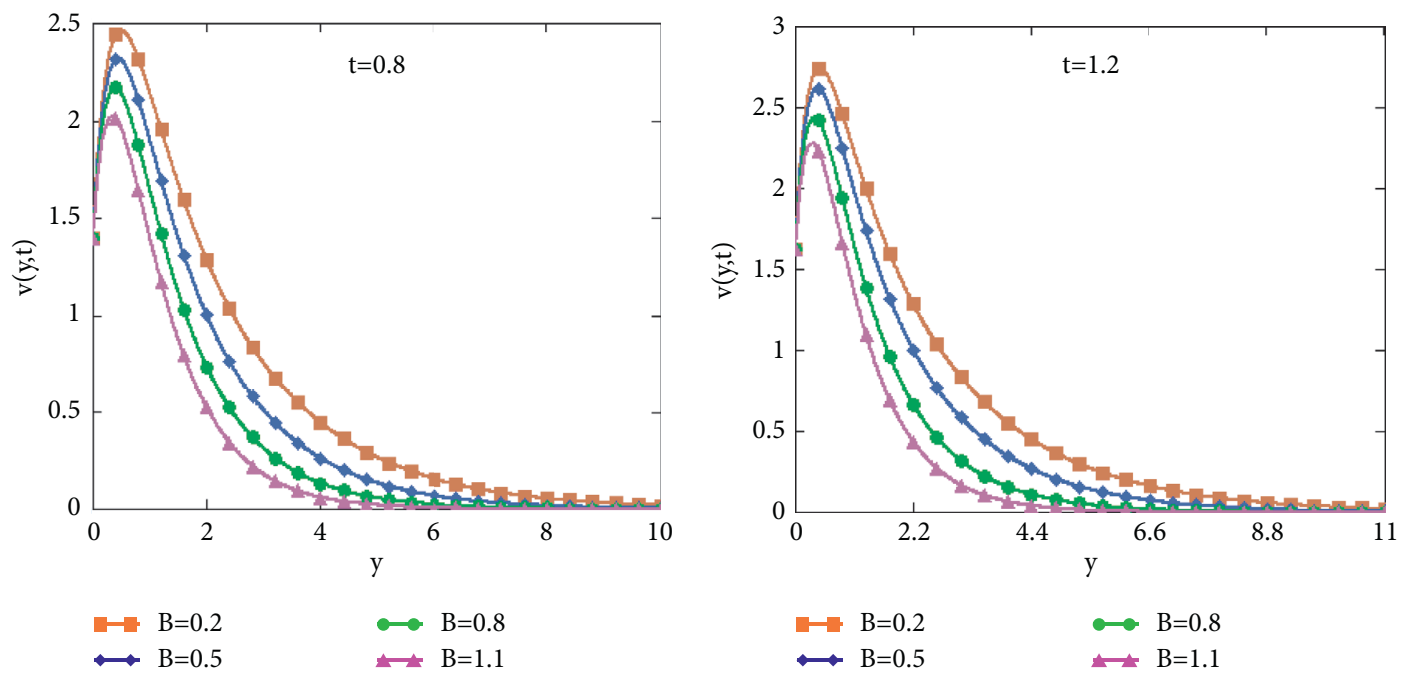

FIGURE 1: Velocity diagram $v(y, t)$ for various values of Brinkman parameter $B$ at $R=1.4, Q=0.4, \mathrm{Gr}=9, \mathrm{Gm}=6, M=.85$, $\alpha=\beta=\gamma=0.5, \mathrm{Sc}=4.5$, and $\operatorname{Pr}=4.0$.
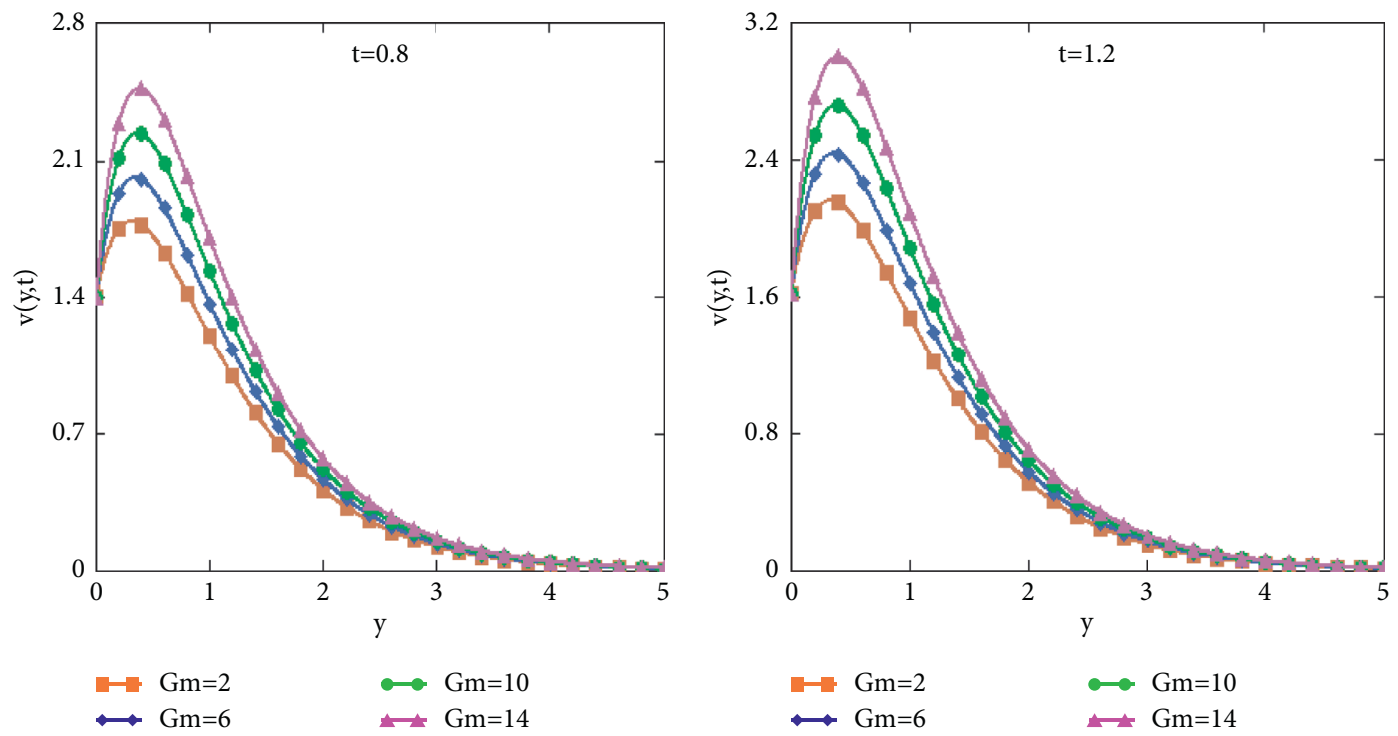

Figure 2: Profiles of velocity $v(y, t)$ for various values of the mass Grashof number $\mathrm{Gm}$ at $R=1.4, \mathrm{Q}=0.4, \mathrm{Gr}=9, M=.85, \mathrm{Sc}=4.5$, $\alpha=\beta=\gamma=0.5, B=0.2$, and $\operatorname{Pr}=4.0$.

$R$ which slows down the velocity distribution. Figure 6 shows the influence of Sc and $\alpha, \beta$, and $\gamma$ on $v(y, t)$. The graph shows that for increasing values of Sc, the diffusion of the molecule increases which reduces the fluid level. However, fluid velocity rises with increasing values of fractional parameters.

The behavior of heat generation $Q$ and $\operatorname{Pr}$ on $T(y, t)$ is displayed in Figure 7. This figure shows that temperature increases with increment in the values of $Q$. Figure 7 indicates the influence of $\operatorname{Pr}$ on temperature $T(y, t)$. Temperature distribution is accelerated with decreasing values of $\operatorname{Pr}$ as shown in the graph. Physically, the increase in $\mathrm{Pr}$ minimizes viscosity which reduces the thermal boundary layer.
The behavior of chemical reaction $R$ and Sc on $C(y, t)$ is shown in Figure 8. The concentration level is accelerated with decreasing $R$ as depicted in the graph. Physically, boundary layer thickness is increased by increasing values of $R$ which slows down the concentration distribution. Figure 8 shows the influence of Sc on $C(y, t)$. The concentration level increases with reducing values of $S c$ as highlighted in the figure. The graph shows that for increasing values of Sc, the diffusion of molecule increases which reduces the fluid level. Figure 9 shows the comparison of Brinkman-type fractional fluid with Olisa [48]. From the figure, it is concluded that fractional derivative is the best choice to enhance the fluid motion. Figure 9(b) represents that if we take fractional parameters $\beta=\gamma=\alpha \longrightarrow 1, \mathrm{Gm}=F(s)=0$, and $B=0$, the 

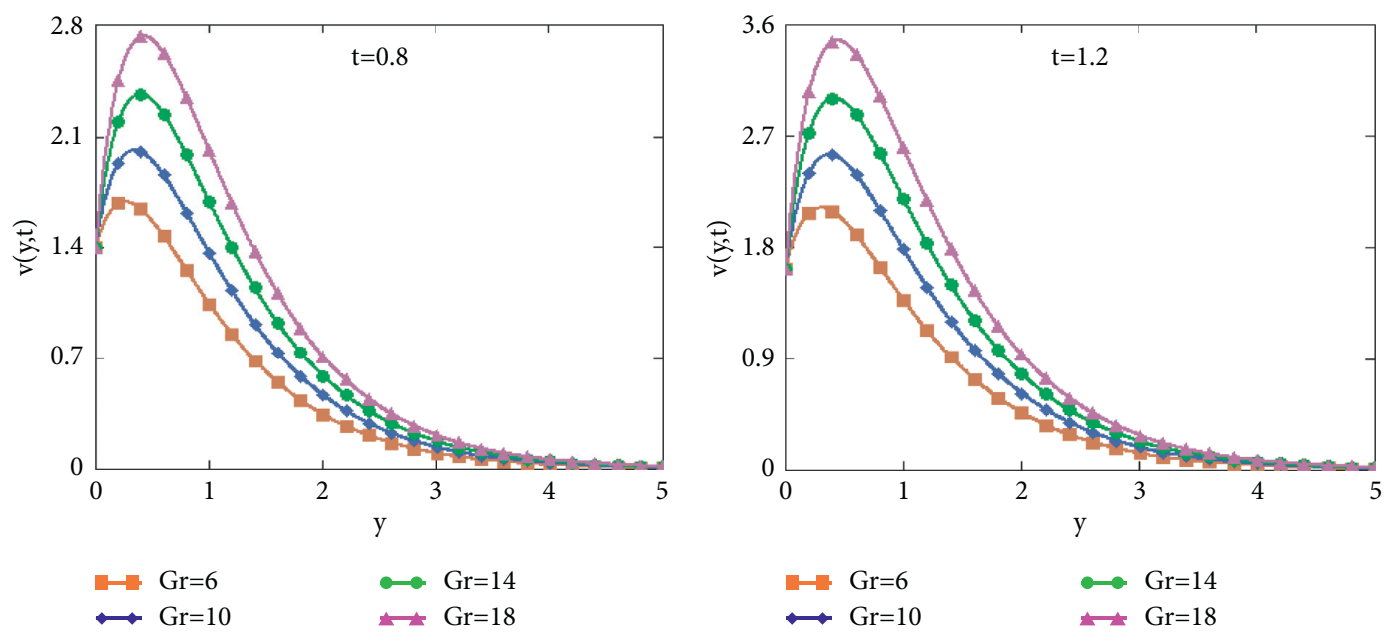

Figure 3: Velocity profile $v(y, t)$ for various values of $\mathrm{Gr}$ at $R=1.4, Q=0.4, \mathrm{Gm}=6, M=.85, \mathrm{Sc}=4.5, \alpha=\beta=\gamma=0.5, B=0.2$, and $\operatorname{Pr}=4.0$.
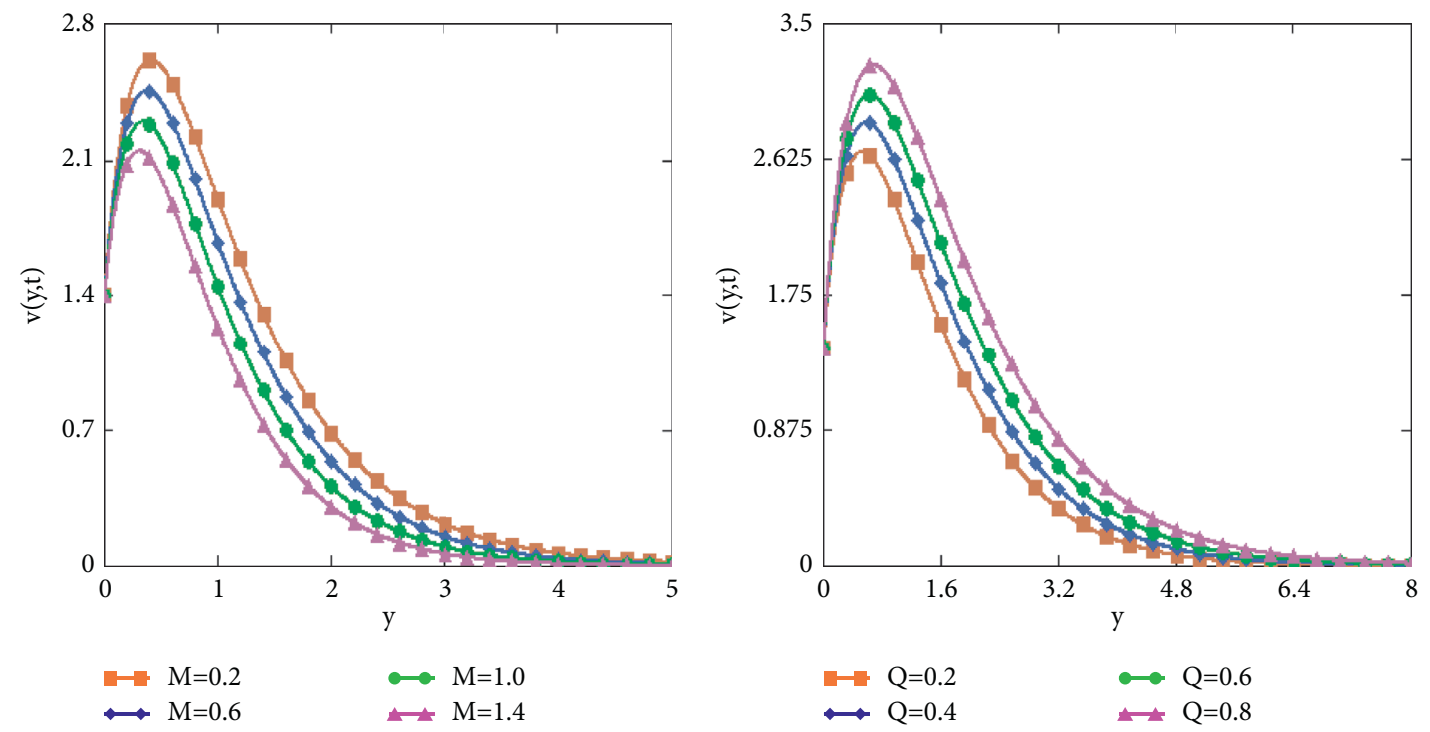

Figure 4: Velocity distribution $v(y, t)$ for distinct values of $M$ and heat generation parameter $Q$ at $R=1.4, \mathrm{Gr}=9, \mathrm{Gm}=6, \mathrm{Sc}=4.5, \alpha=\beta=\gamma=0.5, B=0.2$, and $\operatorname{Pr}=4.0$.
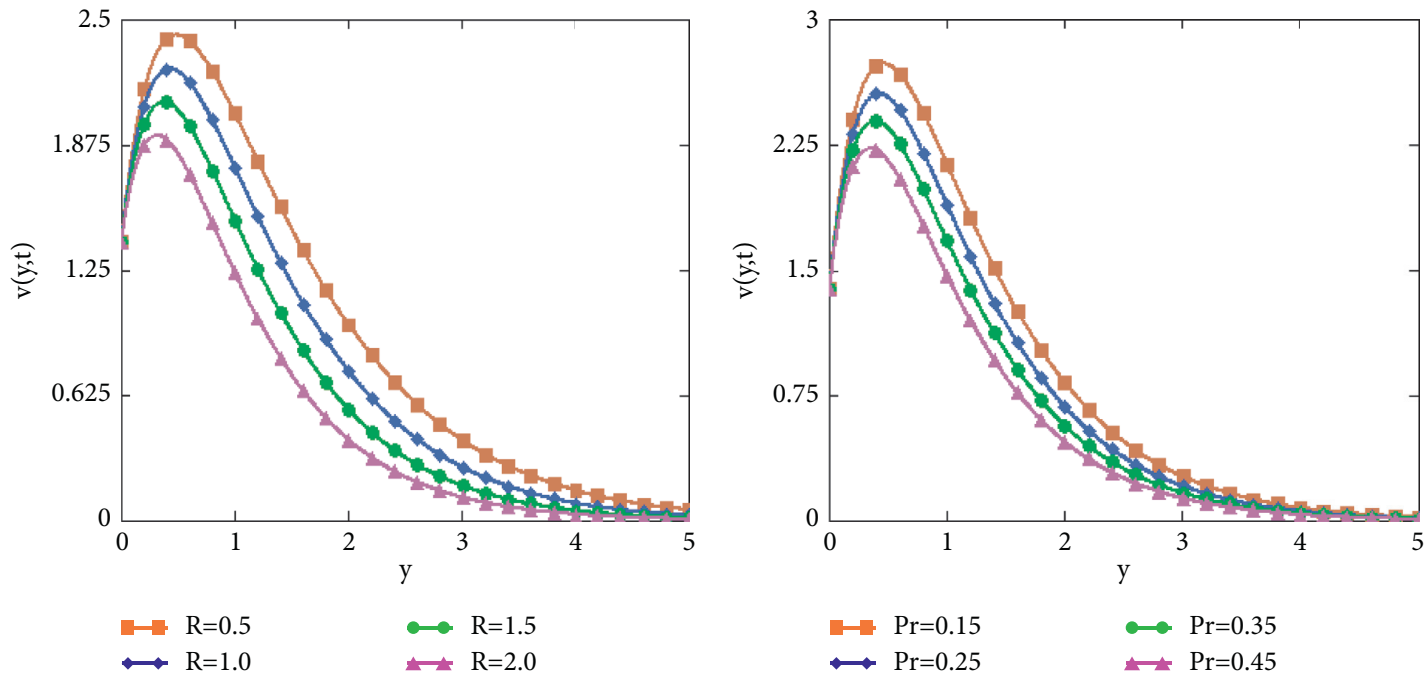

Figure 5: Velocity diagram $v(y, t)$ for distinct values of chemical reaction parameter $R$ and $\operatorname{Pr}$ at $Q=0.4, \mathrm{Gr}=9, \mathrm{Gm}=6, M=.85, \mathrm{Sc}=4.5$, $\alpha=\beta=\gamma=0.5$, and $B=0.2$. 

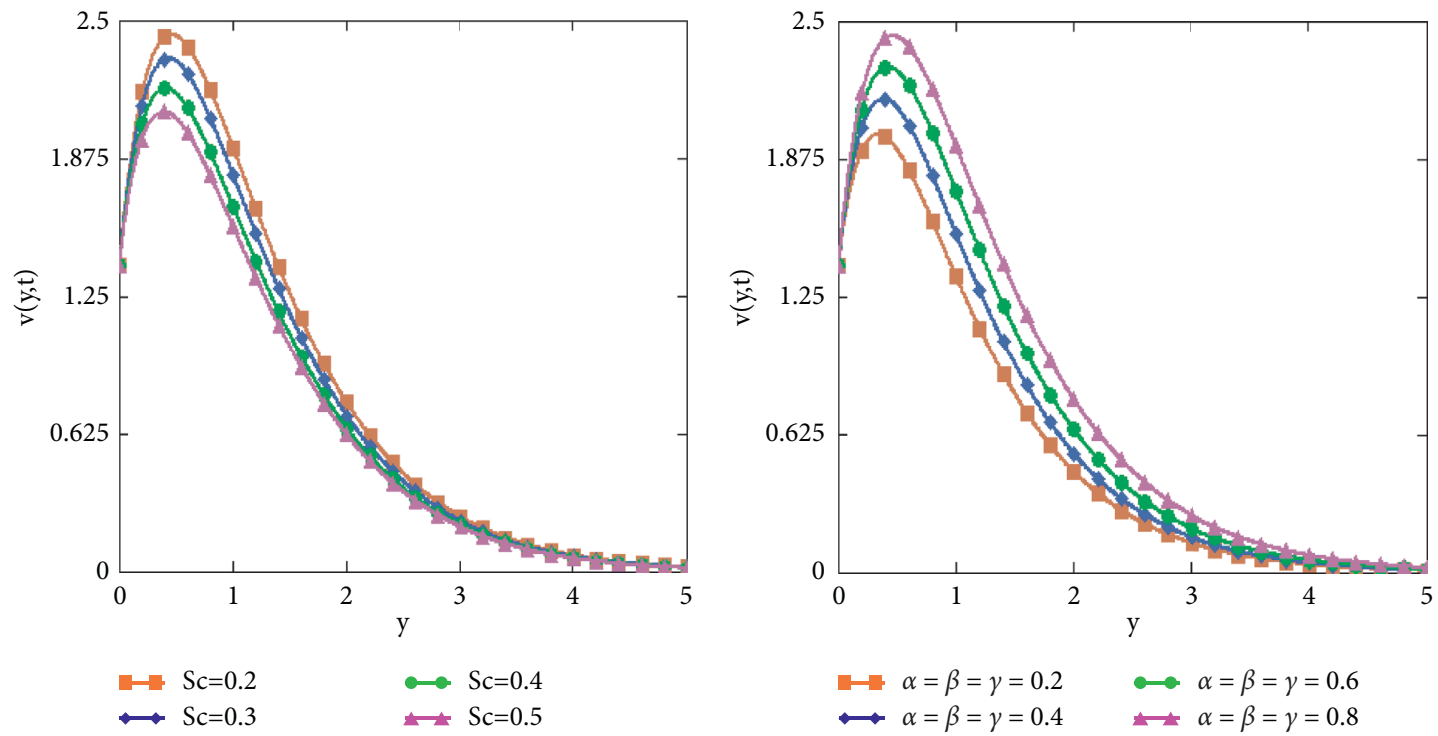

Figure 6: $v(y, t)$ for distinct values of $\mathrm{Sc}$ and fractional parameters at $R=1.4, \mathrm{Q}=0.4, \mathrm{Gr}=9, \mathrm{Gm}=6, M=.85, B=0.2$, and $\mathrm{Pr}=4.0$.
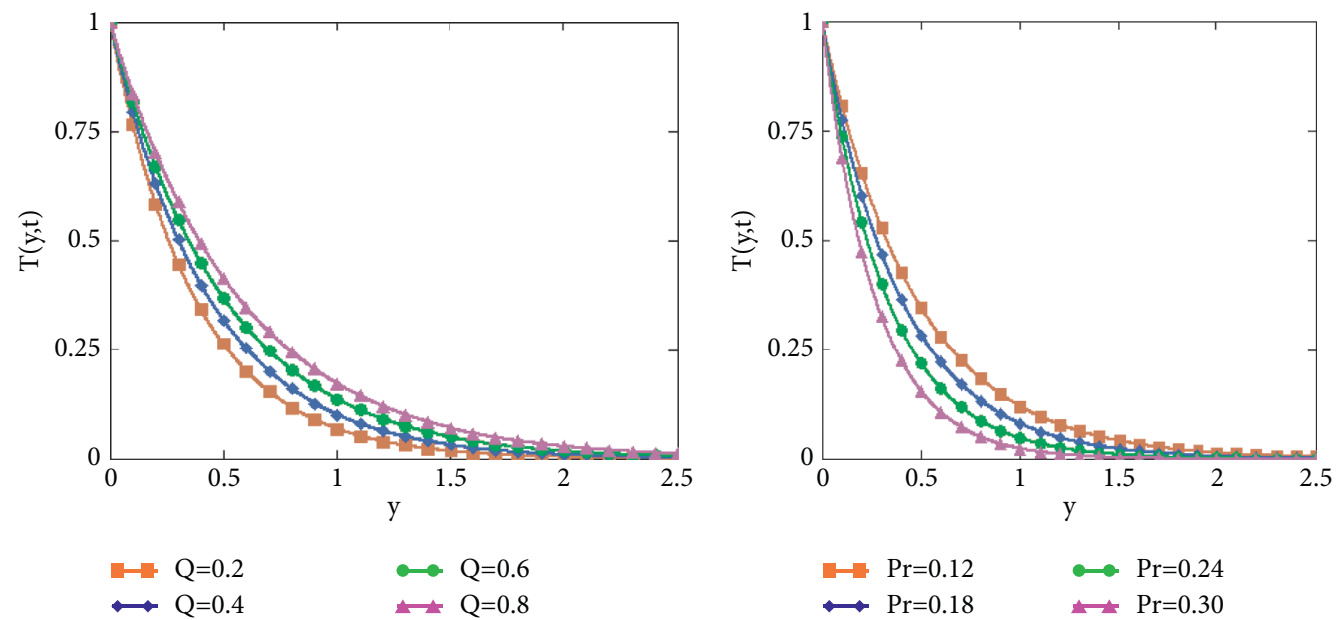

Figure 7: Variation in temperature profile $T(y, t)$ for various values of heat generation parameter $Q$ and $\operatorname{Pr}$ at $\gamma=0.5$.
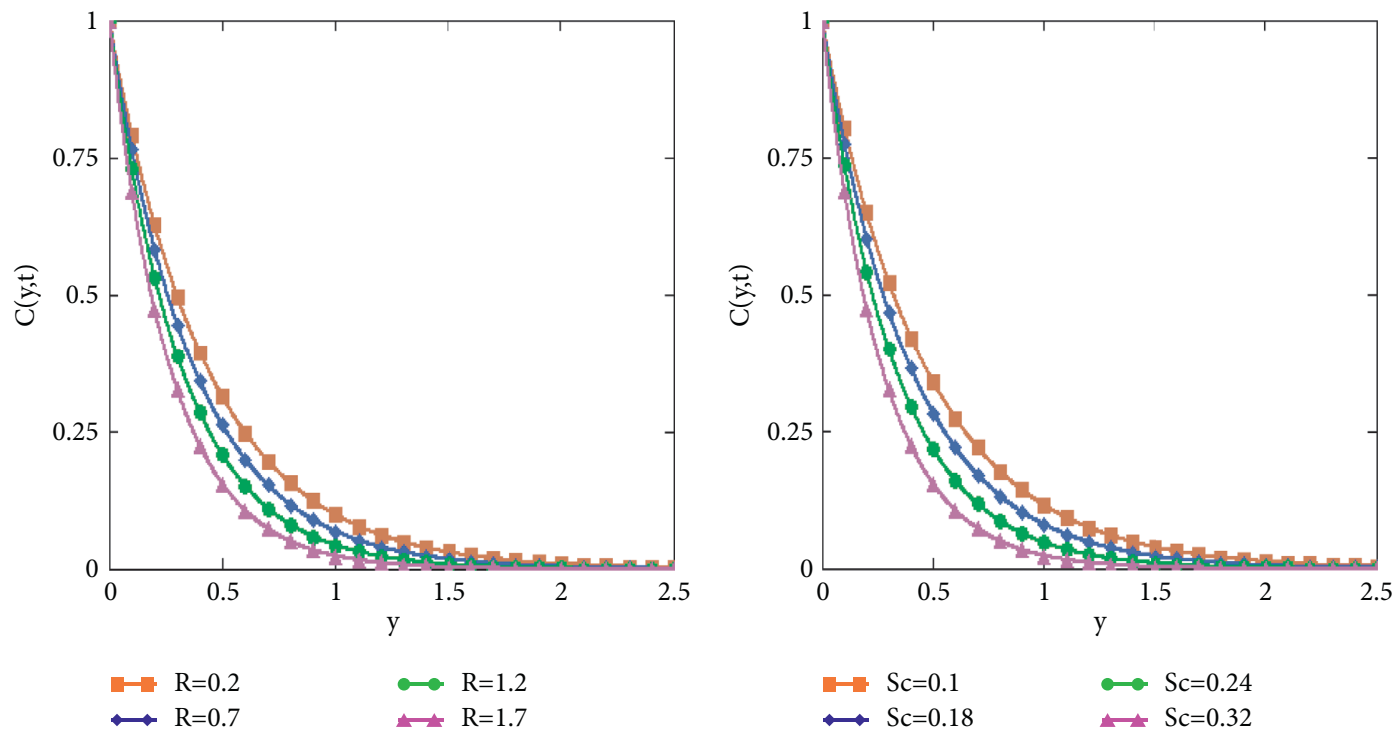

Figure 8: $C(y, t)$ for distinct values of chemical reaction parameter $R$ and Sc at $\alpha=0.5$. 

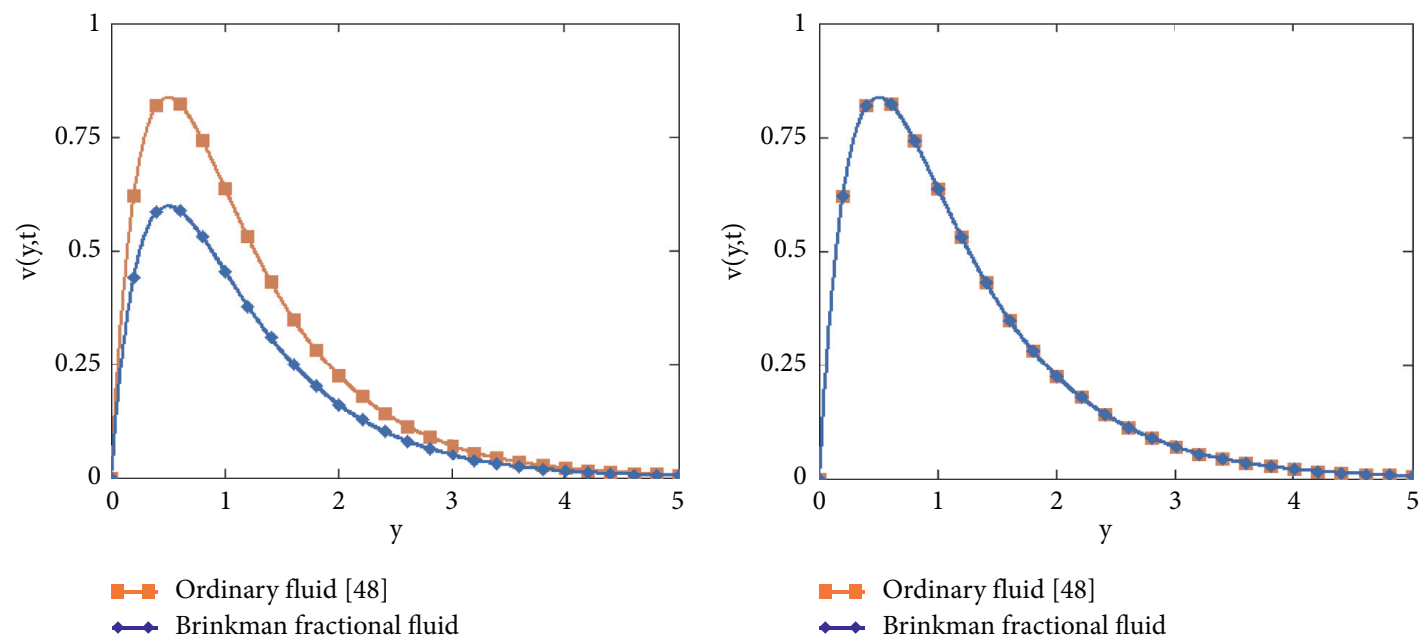

FIgURE 9: Velocity distribution for comparison of our work with Olisa [48].
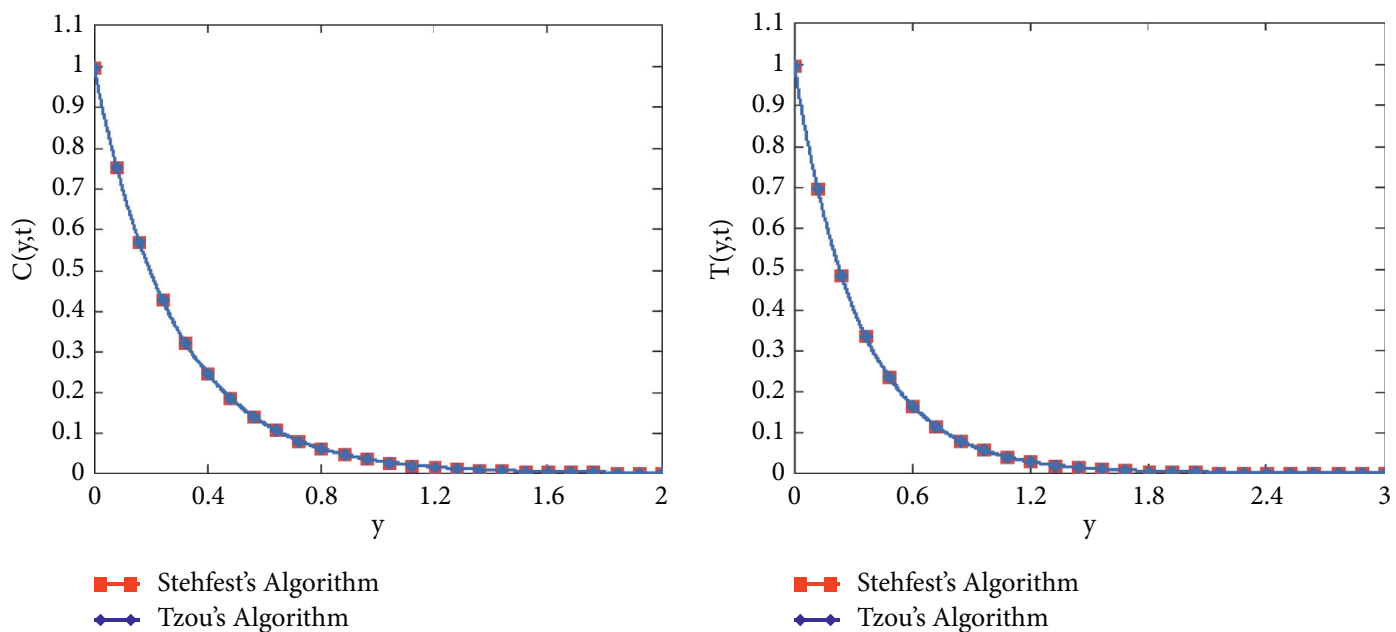

- Stehfest's Algorithm

Tzou's Algorithm

$\rightarrow$ Tzou's Algorithm

FIgURE 10: Concentrations and temperatures obtained by Stehfest's and Tzou's algorithms.

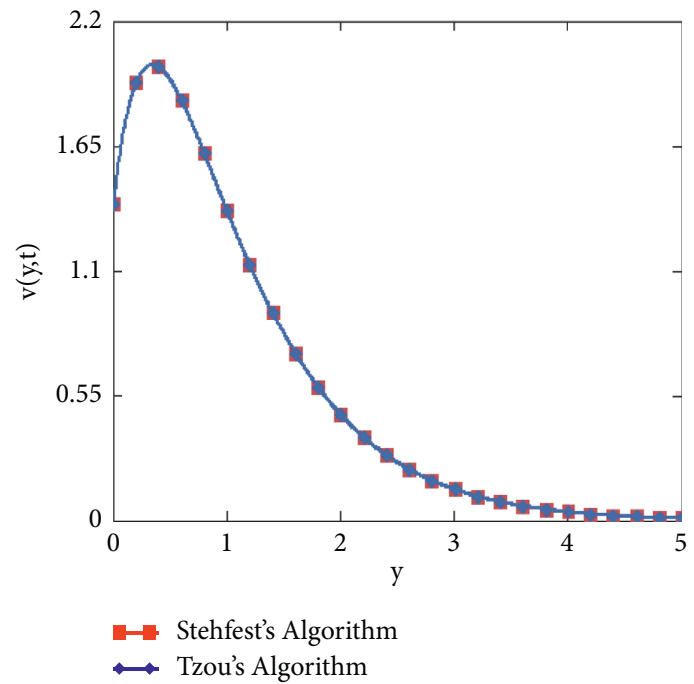

Figure 11: Velocity profile obtained by Stehfest's and Tzou's algorithms. 
fluid profiles are identical which shows the authenticity of the present work. Figure 10 represents the validity of inversion algorithms for concentration and temperature profiles. The overlapping velocity profiles show the validity of inversion algorithms as shown in Figure 11.

\section{Conclusion}

Solution of free convection magnetohydrodynamic flow of Brinkman-type fluid has been obtained via Laplace transform. Different parameters used in the model are plotted and discussed. The model is solved with a fractional derivative known as Caputo fractional derivative.

Here are the main points which have been summarized for this model:

(i) Velocity distribution retards with decreasing values of fractional parameter

(ii) Thermal buoyancy forces lead to accelerate the $v(y, t)$

(iii) The $v(y, t)$ decreases as magnetic parameter, chemical reaction parameter, Prandtl number, and Sc increases

(iv) The Brinkman parameter is a decreasing function of velocity field

(v) The larger values of $Q$ increased the $T(y, t)$

(vi) The larger values of Pr reduced the $T(y, t)$

(vii) The concentration level is a decreasing function of $\mathrm{Sc}$

(viii) The smaller values of $R$ reduce the concentration profile

(ix) Caputo fractional derivative is the best choice to enhance the fluid motion as compared to ordinary fluid

\section{Data Availability}

All used data are included within the manuscript.

\section{Conflicts of Interest}

The authors declare that they have no conflicts of interest.

\section{References}

[1] R. M. Swamy, P. Ganesan, and V. M. Soundalgekar, "Heat and mass transfer effects on flow past an impulsively started vertical plate," Acta Mechanica, vol. 146, no. 1-2, pp. 1-8, 2001.

[2] R. C. Chaudhary and A. Jain, "Combined heat and mass transfer effects on MHD free convection flow past an oscillating plate embedded in porous medium," Romanian Journal of Physics, vol. 52, no. 5-7, pp. 505-524, 2007.

[3] M. Sivaiah, A. S. Nagarajan, and P. S. Reddy, "Heat and mass transfer effects on MHD free convective flow past a vertical porous plate," Journal of Computational Mathematics, vol. 2, no. 2, pp. 14-21, 2009.

[4] K. Das and S. Jana, "Heat and mass transfer effects on unsteady MHD free convection flow near a moving vertical plate in porous medium," Bulletin of Society of Mathematicians Banja Luka, vol. 17, no. 10, pp. 15-32, 2010.

[5] H. R. Kataria and H. R. Patel, "Radiation and chemical reaction effects on MHD Casson fluid flow past an oscillating vertical plate embedded in porous medium," Alexandria Engineering Journal, vol. 55, no. 1, pp. 583-595, 2016.

[6] A. J. Chamkha, "Thermal radiation and buoyancy effects on hydromagnetic flow over an accelerating permeable surface with heat source or sink," International Journal of Engineering Science, vol. 38, no. 15, pp. 1699-1712, 2000.

[7] A. J. Chamkha, "Unsteady MHD convective heat and mass transfer past a semi-infinite vertical permeable moving plate with heat absorption," International Journal of Engineering Science, vol. 42, no. 2, pp. 217-230, 2004.

[8] Y. J. Kim, "Unsteady MHD convection flow of polar fluids past a vertical moving porous plate in a porous medium," International Journal of Heat and Mass Transfer, vol. 44, no. 15, pp. 2791-2799, 2001.

[9] M. M. Rahman and M. A. Sattar, "Magnetohydrodynamic convective flow of a micropolar fluid past a continuously moving vertical porous plate in the presence of heat generation/absorption," Journal of Heat Transfer, vol. 128, no. 2, pp. 142-152, 2006.

[10] V. Rajesh and S. V. K. Varma, "Radiation effects on MHD flow through a porous medium with variable temperature or variable mass diffusion," International Journal of Applied Mathematics and Mechanics, vol. 6, no. 1, pp. 39-57, 2010.

[11] M. A. Sattar, "Free convection and mass transfer flow through a porous medium past an infinite vertical porous plate with time dependent temperature and concentration," International Journal of Pure and Applied Mathematics, vol. 23, pp. 759-766, 1994.

[12] D. A. S. Rees and I. Pop, "Free convection induced by a vertical wavy surface with uniform heat flux in a porous medium," Journal of Heat Transfer, vol. 117, no. 2, pp. 547-550, 1995.

[13] M. Acharya, G. C. Dash, and I. P. Singh, "Magnetic field effects on the free convection and mass transfer flow through porous medium with constant suction and constant heat flux," Indian Journal of Pure and Applied Mathematics, vol. 31, no. 1, pp. 1-18, 2000.

[14] C. Y. Ching-Yang Cheng, "Natural convection heat and mass transfer near a vertical wavy surface with constant wall temperature and concentration in a porous medium," International Communications in Heat and Mass Transfer, vol. 27, no. 8, pp. 1143-1154, 2000.

[15] A. Khan, I. Khan, F. Ali, and S. Shafie, "Effects of wall shear stress MHD conjugate flow over an inclined plate in a porous medium with ramped wall temperature," Mathematical Problems in Engineering, vol. 4, pp. 62-73, 2014.

[16] V. Rajesh and S. V. K. Varma, "Heat source effects on MHD flow past an exponentially accelerated vertical plate with variable temperature through a porous medium," International Journal of Applied Mathematics and Mechanics, vol. 6, no. 12, pp. 68-78, 2010.

[17] R. C. Chaudhary and A. Jain, "An exact solution of magnetohydrodynamic convection flow past an accelerated surface embedded in a porous medium," International Journal of Heat and Mass Transfer, vol. 53, no. 7, pp. 1609-1611, 2010.

[18] K. Das, "Exact solution of MHD free convection flow and mass transfer near a moving vertical plate in presence of thermal radiation," African Journal of Mathematical Physics, vol. 8, pp. 29-41, 2010. 
[19] D. Pal and B. Talukdar, "Buoyancy and chemical reaction effects on MHD mixed convection heat and mass transfer in a porous medium with thermal radiation and ohmic heating," Communications in Nonlinear Science and Numerical Simulation, vol. 15, no. 10, pp. 2878-2893, 2010.

[20] G. S. Seth, M. S. Ansari, and R. Nandkeolyar, "MHD natural convection flow with radiative heat transfer past an impulsively moving plate with ramped wall temperature," Heat and Mass Transfer, vol. 47, no. 5, pp. 551-561, 2011.

[21] A. Khalid, I. Khan, and S. Shafie, "Exact solutions for free convection flow of nano fluids with ramped wall temperature," European Physical Journal Plus, vol. 130, pp. 243-255, 2015.

[22] M. A. Seddeek, A. A. Darwish, and M. S. Abdelmeguid, "Effects of chemical reaction and variable viscosity on hydromagnetic mixed convection heat and mass transfer for hiemenz flow through porous media with radiation," Communications in Nonlinear Science and Numerical Simulation, vol. 12, no. 2, pp. 195-213, 2007.

[23] N. A. Shah, A. A. Zafar, and S. Akhtar, "General solution for MHD-free convection flow over a vertical plate with ramped wall temperature and chemical reaction," Arabian Journal of Mathematics, vol. 7, no. 1, pp. 49-60, 2018.

[24] G. S. Seth, A. K. Singha, and R. Sharma, "MHD natural convection flow with hall effects, radiation and heat absorption over an exponentially accelerated vertical plate with ramped temperature," Indian Journal of Scientific Research and Technology, vol. 5, pp. 10-22, 2015.

[25] S. Shateyi and S. Motsa, "Unsteady magnetohydrodynamic convective heat and mass transfer past an infinite vertical plate in a porous medium with thermal radiation, heat generation/ absorption and chemical reaction," Advanced Topics in Mass Transfer, vol. 1, pp. 145-162, 2011.

[26] A. R. M. Kasim, N. F. Mohammad, and S. Shafie, "Unsteady MHD mixed convection flow with heat and mass transfer over a vertical plate in a micropolar fluid-saturated porous medium," Journal of Applied Science and Engineering, vol. 16, no. 2, pp. 141-150, 2013.

[27] F. Ali, I. Khan, S. Shafie, and N. Musthapa, "Heat and mass transfer with free convection MHD flow past a vertical plate embedded in a porous medium," Mathematical Problems in Engineering, vol. 2013, pp. 1-13, 2013.

[28] Z. A. Khan, S. U. Haq, T. S. Khan, I. Khan, and I. Tlili, "Unsteady MHD flow of a Brinkman type fluid between two side walls perpendicular to an infinite plate," Results in Physics, vol. 9, pp. 1602-1608, 2018.

[29] F. Ali, M. Gohar, and I. Khan, "MHD flow of water-based Brinkman type nanofluid over a vertical plate embedded in a porous medium with variable surface velocity, temperature and concentration," Journal of Molecular Liquids, vol. 223, pp. 412-419, 2016.

[30] M. Sheikholeslami, H. R. Kataria, and A. S. Mittal, "Effect of thermal diffusion and heat-generation on MHD nanofluid flow past an oscillating vertical plate through porous medium," Journal of Molecular Liquids, vol. 257, pp. 12-25, 2018.

[31] H. R. Kataria and A. S. Mittal, "Velocity, mass and temperature analysis of gravity-driven convection nanofluid flow past an oscillating vertical plate in the presence of magnetic field in a porous medium," Applied Thermal Engineering, vol. 110, pp. 864-874, 2017.

[32] H. R. Patel, A. S. Mittal, and R. R. Darji, "MHD flow of micropolar nanofluid over a streching/shrinking sheet considering radiation," Journal Comunication in heat and mass transfer, vol. 51, pp. 45-69, 2019.
[33] H. R. Kataria and A. S. Mittal, "Mathematical model for velocity and temperature of gravity-driven convective optically thick nanofluid flow past an oscillating vertical plate in presence of magnetic field and radiation," Journal of the Nigerian Mathematical Society, vol. 34, no. 3, pp. 303-317, 2015.

[34] A. S. Mittal and H. R. Kataria, "Three dimensional Cuo-Water nanofluid flow considering Brownian motion in presence of radiation," Karbala International Journal of Modern Science, vol. 4, no. 3, pp. 275-286, 2018.

[35] M. Sheikholeslami, H. R. Kataria, and A. S. Mittal, "Radiation effects on heat transfer of three dimensional nanofluid flow considering thermal interfacial resistance and micro mixing in suspensions," Chinese Journal of Physics, vol. 55, no. 6, pp. 2254-2272, 2017.

[36] H. R. Patel, A. S. Mittal, and R. R. Darji, "Mixed convection micropolar ferrofluid flow with viscous dissipation, joule heating and convective boundary conditions," Journal Comunication in heat and mass transfer, vol. 110, pp. 864-874, 2017.

[37] A. S. Mittal and H. R. Kataria, "Infulence of thermophoresis and Brownian motion on mixed convection two dimensional MHD Casson fluid flow with non linear radiation and heat generation," Physica A: Statistical Mechanics and its Applications, vol. 537, 2020.

[38] H. R. Kataria, "Analysis of casson nanofluid flow in presence of magnetic field and radiation," Journal of mathematics today, vol. 33, pp. 99-120, 2017.

[39] M. S. Hashemi, M. Inc, B. Kilic, and A. Akgül, "On solitons and invariant solutions of the Magneto-electro-elastic circular rod," Waves in Random and Complex Media, vol. 26, no. 3, pp. 259-271, 2016.

[40] M. S. Hashemi, "Constructing a new geometric numerical integration method to the nonlinear heat transfer equations," Communications in Nonlinear Science and Numerical Simulation, vol. 22, no. 1-3, pp. 990-1001, 2015.

[41] M. S. Hashemi, A. Atangana, and S. Hajikhah, "Solving fractional pantograph delay equations by an effective computational method," Mathematics and Computers in Simulation, vol. 177, pp. 295-305, 2020.

[42] M. S. Hashemi, M. Inc, and A. Yusuf, "On three dimensional variable order time fractional chaotic system with nonsingular Kernal," Journal of Chaos, Solitons and Fractals, vol. 133, 2020.

[43] G. S. Blair and J. Caffyn, "Significance of power law relation in rheology," Nature, vol. 155, pp. 171-172, 1955.

[44] Y. Povstenko, Fractional Thermoelasticity, Solid Mechanics and its Applications, Springer International Publishing, Berlin, Germany, 2015.

[45] J. Hristov, Derivatives with Non-Singular Kernals, from the Caputo-Fabrizo Definition and Beyond, Appraising Analysis with Emphasis on Diffusion Model, Frontiers in Fractional Calculas, Bentham Science Publishers, Sharjah, UAE, 1st edition, 2017.

[46] D. Y. Tzou, Macro to Microscale Heat transfer: the Lagging Behavior, pp. 01-339, Taylor and Francis, Washington, DC, USA, 1997.

[47] H. Stehfest, "Algorithm 368: numerical inversion of Laplace transforms [D5]," Communications of the ACM, vol. 13, no. 1, pp. 47-49, 1970.

[48] J. D. Olisa, "Transient laminar MHD free convective heat transfer past a vertical plate with heat generation," International Journal of Engineering Science, vol. 6, no. 4, pp. 08-13, 2017. 